\title{
Resultados de aprendizagem no quadro do ensino superior português: definição e medida
}

\section{Defining and measuring learning outcomes in Portuguese Higher Education}

\author{
Diana Dias*, Diana Soares*, Leandro S. Almeida**, Claisy Marinho-Araújo***, Joaquim Ferreira **** \\ * Universidade Europeia, **Universidade do Minho, ${ }^{* * *}$ Universidade de Brasília, ${ }^{* * * *}$ Universidade de Coimbra
}

\begin{abstract}
Resumen
Os resultados de aprendizagem assumem-se como importante ferramenta no quadro do Ensino Superior, já que permitem descrever os conhecimentos e competências que se espera que os estudantes demonstrem no final da sua formação. Este estudo descreve o nível de implementação, dos resultados de aprendizagem no contexto nacional, explorando quais as competências mais valorizadas nos perfis de saída dos seus diplomados $\left(1^{\circ}, 2^{\circ}\right.$ e $3^{\circ}$ ciclos $)$. Partindo de uma metodologia mista, foi elaborada uma matriz de análise, validada teórica e empiricamente. Esta taxonomia integra competências específicas à área científica e um conjunto alargado de competências transversais, possibilitando uma leitura compreensiva dos resultados de aprendizagem.

Palabras clave: resultados de aprendizagem,
\end{abstract} competências, Ensino Superior.

\begin{abstract}
The learning outcomes become an important tool in the Higher Education system, since they allow to describe the knowledge and skills that students are expected to acquire at the end of their training. This study intends to evaluate the level of implementation of learning outcomes in the national context, exploring which competences are most valued in the professional profiles of their graduates. Based on a mixed methodology, a matrix of analysis was elaborated, and was validated both theoretical and empirically. This taxonomy integrates discipline-specific competences and a wide range of transversal competences, allowing a comprehensive reading of RA. Keywords: learning outcomes, competences, Higher Education
\end{abstract}

\section{Introdução}

O ensino superior em Portugal (e na Europa) tem sofrido, ao longo dos últimos anos, transformações significativas, muitas delas em resposta aos desafios colocados pelo Processo de Bolonha (Dias \& Amaral, 2014). Um desses desafios remete para a promoção da garantia de qualidade entre os sistemas europeus de ensino superior, com base na sua comparabilidade curricular, em termos de estrutura, programas de estudo e estratégias pedagógicas. Por conseguinte, as metodologias de avaliação, os procedimentos de conceção dos currículos, os conteúdos científicos e os resultados da aprendizagem (RA) devem estar alinhados, permitindo a comparação, não só entre os programas de estudo, mas também entre instituições de ensino superior (Dias \& Amaral, 2014). A definição de um "Quadro de Qualificação do Ensino Superior Europeu", a ser adotado pelos países europeus, pode ser entendido como um exemplo claro desta exigência ao nível da comparabilidade e transparência entre os sistemas educativos (Veiga, Rosa, Dias, \& Amaral, 2013). Cada nível de qualificação define-se em termos do que se espera que o estudante conheça, compreenda e seja capaz de desempenhar no final de um período de formação (Dias \& Amaral, 2014). Ou seja, cada qualificação integra um conjunto específico de RA (learning outcomes), que acaba por corresponder ao perfil de competências esperadas para aquela formação/ciclo de estudos (Dias \& Soares, 2017). Tendo como objetivo principal a análise dos RA definidos nos programas de estudo e currículos das Instituições portuguesas de Ensino Superior, foi necessário, numa primeira etapa, elaborar uma matriz de análise que possibilitasse captar a multiplicidade de dimensões inerentes a este conceito. A elaboração desta matriz é apresentada neste artigo, enfatizando as principais convergências e divergências entre abordagens teóricas e processuais e o extenso conjunto de competências técnicas específicas e genéricas que se podem entender como categorias dos RA.

\section{$O$ conceito de resultados de aprendizagem}

$O$ conceito de RA resulta de uma abordagem relativamente inovadora no quadro das políticas educativas na Europa (Stanley, 2015), assumindo-se "um elemento fundamental da reforma educacional de Bolonha" (Adam, 2006, p. 3). A primeira menção surge no Comunicado de Berlim (2003). Através deste documento, os Estados membros da União Europeia foram encorajados a elaborar um quadro nacional de qualificações, comparável e compatível com os restantes sistemas de Ensino Superior dos diversos países. O objetivo final seria criar um quadro abrangente de qualificações aplicável a todos os países europeus, que deveriam ser descritas em termos de 
carga de trabalho, nível, RA, habilidades e perfil de saída profissional.

Da mesma forma, e conforme sugerido pelos Descritores de Dublin, a cada grau académico correspondem diferentes RA (Joint Quality Initiative informal group, 2004). A cada nível de qualificação, as competências necessárias tornam-se, progressivamente, mais complexas. Por exemplo, as competências de comunicação tornam-se mais exigentes à medida que o nível de qualificação aumenta. Se no $1^{\circ}$ ciclo de estudos (licenciatura em Portugal), se espera que os estudantes "tenham a capacidade de (...) interpretar dados relevantes para informar julgamentos que incluam reflexão sobre questões sociais, científicas ou éticas relevantes", no $2 .^{\circ}$ ciclo de estudo (mestrado), espera-se que "tenham a capacidade de integrar o conhecimento e refletir sobre as responsabilidades sociais e éticas ligadas à aplicação de seus conhecimentos e julgamentos"; finalmente, no $3 .^{\circ}$ ciclo, os estudantes devem já "ser capazes de promover contextos, avanços tecnológicos, sociais ou culturais em uma sociedade baseada no conhecimento". Por outro lado, e no que diz respeito às dimensões de "conhecimento e compreensão" e "aplicação de conhecimento e compreensão", espera-se que um estudante de $1 .^{\circ}$ ciclo aplique conhecimento através da elaboração de argumentos, enquanto se espera que um estudante de $2 .^{\circ}$ ciclo resolva problemas em novos contextos desconhecidos, e um estudante de $3 .^{\circ}$ ciclo seja capaz de conceber, projetar e adaptar um processo de pesquisa. (Joint Quality Initiative informal group, 2004).

Nesta perspetiva da educação baseada em RA, a ênfase é colocada no que se espera que um estudante adquira em termos de conhecimentos, habilidades e competências, em função de cada nível de qualificação (Gallavara, Hreinsson, Kajaste, \& Zadeh, 2008; Tissot, 2008). Da mesma forma, os RA devem explicitar, não só o que se espera que os estudantes venham a saber, como também os critérios que serão utilizados para a sua avaliação e monitorização (Adam, 2004; Moon, 2004; Wiliam, 2010). Desta forma, esta abordagem permite aos estudantes identificar com antecedência o que é esperado que eles conheçam, compreendam e realizem, no decurso da sua formação, bem como os critérios de avaliação que serão utilizados.

\section{(In)Definições de RA}

A literatura em redor desta temática oferece uma grande diversidade de definições e perspetivas sobre o conceito e dimensões inerentes aos RA, o que acaba por torná-lo ambíguo e pouco claro (Aamodt \& Hovdhaugen, 2008; Adam, 2008; Scott, 2011). No Quadro Europeu de Qualificações (European Commission, 2008), os RA são definidos como a afirmação do que um estudante entende e é capaz de fazer na conclusão de determinada formação. Da mesma forma, segundo o Centro Europeu para o Desenvolvimento da Formação Profissional (CEDEFOP, 2008, 2011), os RA incorporam um conjunto de conhecimentos, aptidões e competências. Especificamente, os conhecimentos correspondem ao resultado de uma assimilação de informações, através da aprendizagem, o que inclui factos, princípios, teorias e práticas relacionadas com um campo de trabalho ou estudo. Por sua vez, a capacidade de aplicar conhecimento e usar know-how para completar tarefas e resolver problemas é descrita como uma aptidão. Por fim, a competência remete para a capacidade comprovada de utilizar conhecimentos, aptidões e habilidades pessoais, sociais e/ou metodológicas, em situações de trabalho ou de estudo e no desenvolvimento profissional e pessoal. Além destas dimensões, os RA referem-se a valores, crenças e atitudes a desenvolver pelos estudantes (Werquin, 2012).

Noutros estudos, por exemplo, no "Assessment of Higher Education Learning Outcomes" - AHELO (Tremblay, Lalancette, Roseveare, Dias, \& Amaral, 2013), os RA foram definidos enquanto a combinação de habilidades genéricas e habilidades específicas inerentes à área científica. O pensamento crítico, o conhecimento disciplinar, a resolução de problemas, o trabalho em equipa, a comunicação, as habilidades profissionais, a ética e os valores cívicos, a criatividade e a aprendizagem ao longo da vida são exemplos das dimensões mais significativas de RA.

Também os projetos REFLEX (Allen \& Van der Velden, 2005) e TUNING (Gonzalez \& Wagenaar, 2003) são fundamentais para compreender o conceito de RA. De acordo com o Projeto TUNING, os RA são declarações sobre o que se espera que um estudante conheça, compreenda e/ou possa demonstrar após a conclusão de uma formação. Por sua vez, as competências representam uma combinação de conhecimento, compreensão, aptidões e habilidades. Estas competências podem ser específicas a uma determinada área científica ou genéricas e transversais. No projeto TUNING foram diferenciadas três tipos de competências genéricas: i) as competências instrumentais (habilidades cognitivas, habilidades metodológicas, habilidades tecnológicas e habilidades linguísticas); ii) as competências interpessoais (habilidades sociais, de interação social e cooperação); e as iii) as competências sistêmicas (habilidades mais complexas, como a combinação de compreensão, sensibilidade e conhecimento, necessária aquisição prévia de competências instrumentais e interpessoais). Uma lista de 31 competências genéricas foi considerada neste projeto. Algumas delas estão relacionados com a capacidade de: comunicar num segundo idioma; aprender ao longo da vida; comunicação oral e escrita; autoavaliação; planear e gerir o tempo; de gerar novas ideias; pesquisar, processar e analisar informação; identificar, colocar e resolver problemas; tomar decisões; realizar pesquisas; trabalhar em equipa ou em contextos internacionais; atuar com base em princípios éticos e deontológicos; entre outras.

Da mesma forma, para Adam (2004, 2008), os RA são a "combinação entre conhecimento, habilidades, aptidões, atitudes e entendimentos a serem alcançados pelo estudante" (p.2), podendo ser mais específicas à área de formação ou mais genéricas e transferíveis. 
Segundo Nusche (2008), "o que um estudante sabe ou pode fazer como resultado da aprendizagem" (p. 7) pode ser categorizado em termos de resultados cognitivos e não cognitivos. Os RA de base cognitiva ramificam-se em áreas de conhecimento mais específicos e em processos de pensamento e de resolução de problemas (Shavelson \& Huang, 2003). Como tal, os RA de base cognitiva incluem a aquisição de conhecimentos genéricos e específicos, bem como a aquisição de determinadas habilidades/aptidões, tais como o raciocínio verbal e numérico, o processamento analítico, o pensamento crítico ou a resolução de problemas. Estas habilidades são já transferíveis a diferentes áreas disciplinares e contextos de aprendizagem. Por outro lado, as habilidades não cognitivas referem-se a mudanças nas crenças ou à evolução de certos padrões e valores cívicos (Ewell, 2005). O desenvolvimento psicossocial engloba o desenvolvimento pessoal, nomeadamente o desenvolvimento da identidade e da autoestima, bem como a criação de relações interpessoais. A consciencialização social, a motivação para aprender e o respeito pela diversidade cultural são também exemplos de RA de cariz não cognitivo (Dias \& Soares, 2016; Reason \& Hemer, 2012)

\section{Metodologia}

\section{Procedimentos}

A elaboração da matriz de análise decorreu em duas etapas. Num primeiro momento, realizou-se revisão sistemática da literatura, analisando-se um conjunto significativo de artigos científicos e de relatórios e documentos oficiais, tendo por base os seguintes critérios: i) número de citações; e ii) data de publicação. Após a compilação dos documentos, foram comparadas todas as definições e todas as categorias de RA, com base na semelhança e na divergência entre as perspetivas. Esta etapa resultou numa primeira versão da matriz de análise, teoricamente suportada. Num segundo momento, a matriz de análise foi testada empiricamente. Para tal, selecionou-se uma amostra aleatória de 40 propostas de acreditação de novos ciclos de estudos enviados para a Agência de Avaliação e Acreditação do Ensino Superior (A3ES), desde 2009. A análise de conteúdo incidiu sobre as respostas dadas à questão "RA a adquirir pelos estudantes". Quatro avaliadores, após uma formação específica em metodologias qualitativas, analisaram os mesmos 40 RA, tendo como critério a matriz de análise elaborada na primeira fase. $\mathrm{O}$ acordo de avaliadores foi calculado e, após discussão entre todos os avaliadores, foi elaborada uma segunda versão da matriz de análise. Esta versão foi novamente testada por dois avaliadores, codificando aleatoriamente $20 \mathrm{RA}$. Com base nos resultados do acordo de avaliadores e após validação por especialistas académicos em termos da sua abrangência e diferenciação, chegou-se a versão final da matriz de análise.

\section{Resultados}

A matriz de análise divide-se em duas macro-categorias principais que, por sua vez, se subdivide em várias micro-categorias. Conforme apresentado na figura 1 , os conhecimentos (ou competências técnicas) integram os conhecimentos específicos e os genéricos. Os conhecimentos específicos à área científica podem ser práticos (ex. "conceber, implementar e avaliar um plano de comunicação") ou teóricos (ex. "assimilar informações sobre teorias psicológicas da aprendizagem").

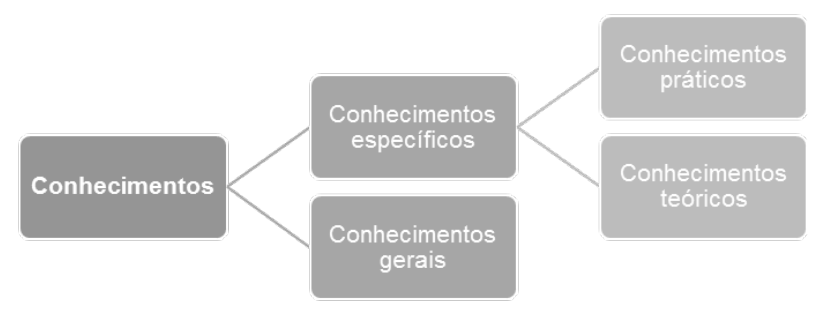

Fig. 1 Matriz de análise dos RA (I)

Por outro lado, as competências genéricas/transversais incorporam um conjunto de 21 micro-categorias (ver figura 2). "Ser capaz de tomar as melhores decisões no processo de desenvolvimento de um produto" é um exemplo de um RA codificado como tomada de decisão. A resolução de problemas está relacionada com a "capacidade de diagnosticar problemas e usar diferentes modelos qualitativos e quantitativos na análise de situações complexas". A "capacidade de planear e organizar o trabalho" é, também, um exemplo de uma micro-dimensão. $\mathrm{O}$ pensamento crítico ("desenvolvimento de capacidades analíticas e críticas") e "a capacidade de comunicar para diferentes audiências, oralmente, por escrito ou numa língua estrangeira" são consideradas três micro-categorias da categoria Comunicação. "Saber gerir dados e informações" e "adotar um papel ativo no uso novas tecnologias", correspondem às categorias Gestão da Informação e Tecnologias de Comunicação de Informação. Por sua vez, o trabalho em equipa compreende duas micro-dimensões: trabalho em equipas internacionais e multidisciplinares. Aqui, "ser capaz de colaborar em equipas com elementos de outras áreas científicas" é um exemplo de RA. O relacionamento interpessoal divide-se em duas dimensões: negociação e gestão de conflitos. A motivação para o desenvolvimento de uma pesquisa rigorosa e a excelência no trabalho, bem como a capacidade para se adaptar a novos contextos e situações também são dimensões-chave desta matriz de análise. As competências ligadas à inovação, empreendedorismo e autonomia também são consideradas. Uma dimensão central é a aprendizagem ao longo da vida, que corresponde ao "desenvolvimento pessoal contínuo ao longo da vida". Por último, um conjunto de quatro dimensões, relacionadas com 
atitudes e competências cívicas, tais como a ética e a deontologia profissional são enfatizadas. "Assumir a responsabilidade pessoal e social pelas suas próprias ações e comportamentos", ou "desenvolver uma consciência cultural " são RA representativos dessas dimensões.

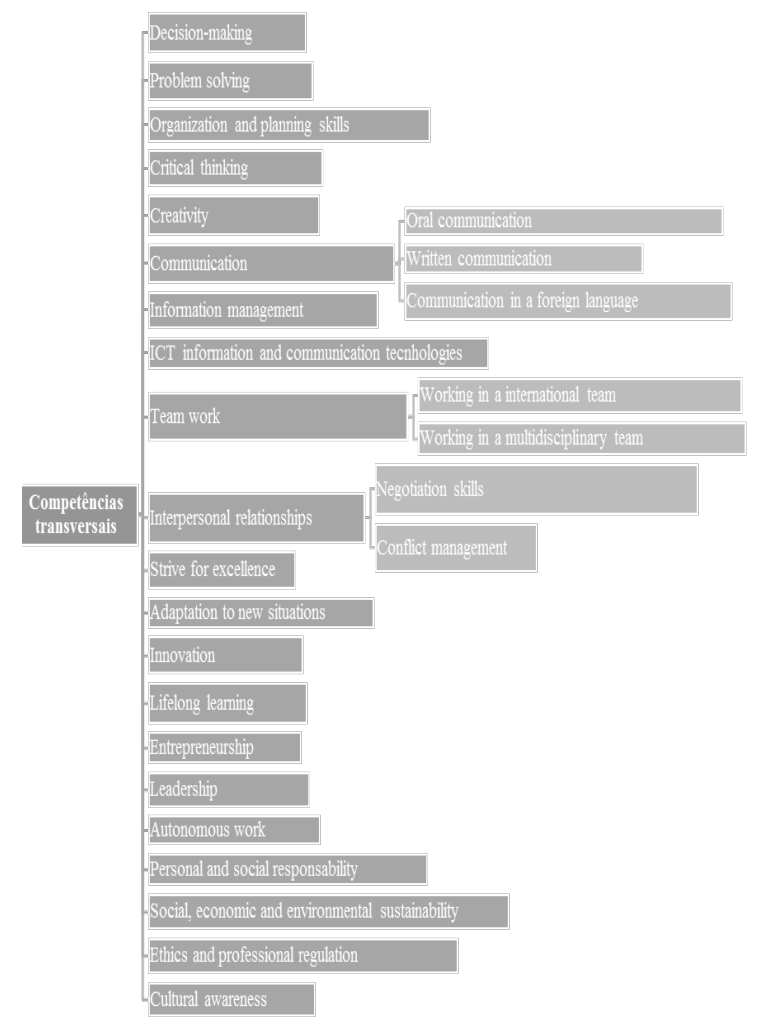

Fig. 1 Matriz de análise dos RA (II)

\section{Discussão e conclusão}

Face à diversidade de abordagens e perspetivas, tanto teóricas como empíricas, sobre a definição e tipologia de RA, torna-se pertinente a definição de uma matriz de análise que possa conciliar as diferentes taxonomias propostas na literatura. Esta aparente polissemia associada ao conceito de RA complexifica esta tarefa, obrigando a encontrar pontos de convergência entre diversas perspetivas de forma a ser possível definir um quadro teórico robusto, abrangente e válido. Neste artigo surge neste esforço, pretendendo apresentar uma matriz de análise capaz de avaliar como os RA são conceptualizados pelas instituições de ensino superior e quais as competências técnicas e transversais que tendem a ser mais valorizadas nos perfis dos futuros graduados.

Com base numa revisão sistemática da literatura, foram analisadas as convergências e divergências entre taxonomias e quadros teóricos. Esta análise resultou na definição de um conjunto de competências específicas (à área científica) e genéricas entendidas como exemplos representativos de RA. Esta tarefa é particularmente complexa se se considerar a ampla diversidade de perspetivas teóricas sobre o tema. Não só é pouco clara a definição de RA (Aamodt \& Hovdhaugen, 2008; Adam, 2008; Scott, 2011), como também o "catálogo" de competências a ele associado tende a variar entre os autores.

Tendo por base uma definição de RA assente na "combinação entre conhecimento, aptidões, habilidades, atitudes a adquirir pelos estudantes" (Adam, 2004, p.2), esta matriz de análise engloba, por isso, as competências inerentes à própria disciplina e as competências transversais mais referenciadas na literatura (Adam, 2008; Gonzalez \& Wagenaar, 2003; Nusche, 2008; OECD, 2013). Um aspeto a destacar remete para a flexibilidade e a abrangência da matriz, definida de forma suficientemente ampla para se adequar a diferentes perspetivas teóricas, permitindo comparações entre investigadores, taxonomias ou configurações de ensino superior (Adam, 2008; Garcia-Aracil \& Van Der Velden, 2008; Nusche, 2008).

Em suma, a metodologia que sustenta a elaboração da matriz de análise de RA integra não só uma revisão sistemática da literatura, na qual uma variedade de documentos institucionais e artigos científicos foi analisada, comparada e contrastada entre si, como também uma vertente mais empírica, baseada no acordo de avaliadores e consulta de juízes. Neste sentido, assumimos que esta matriz se encontra validada teórica e empiricamente.

Um desenvolvimento adicional deste estudo implica a aplicação sistemática desta matriz à análise de conteúdo de RA propostos pelas instituições portuguesas de Ensino Superior no âmbito dos pedidos de acreditação dos seus novos ciclos de estudo e ciclos de estudo em funcionamento, etapa essa que se encontra já a ser concretizada. Uma comparação internacional seria, igualmente, um estudo complementar interessante na validação da matriz de análise apresentada.

\section{Referencias}

Aamodt, P. O., \& Hovdhaugen, E. (2008). Assessing Higher Education learning outcomes as a result of institutional and individual characteristics. Outcomes of Higher Education: Quality relevance and impact. Paris: OECD.

Adam, S. (2004). Using learning outcomes; A consideration of the nature, role, application and implications for European education of employing "learning outcomes" at the local, national and international levels. United Kingdom Bologna Seminar, 1-2 July 2004, Heriot-Watt University (Edinburgh Conference Centre), (July), 30. http://doi.org/Heriot-Watt University

Adam, S. (2006). An introduction to learning outcomes. In F. E., J. Kohler, L. Purser, \& L. Wilson (Eds.), EUA Bologna Handbook. Berlin: Raabe.

Adam, S. (2008). Learning Outcomes based Higher Education: The Scottish experiences. In Paper presented to the Bologna Seminar. Edinburgh.

Allen, J., \& Van der Velden, R. (2005). The Flexible professional in the knowledge society: Conceptual framework of the REFLEX project. Maastricht, The Netherlands. 
CEDEFOP. (2011). When defining learning outcomes in curricula, every learner matters. World (April), 14.

CEDEFOP. (2008). The shift to Learning Outcomes. Office, 56.

Dias, D., \& Amaral, A. (2014). Assessment of Higher Education learning outcomes (AHELO): Feasibility Study. In M. J. Rosa \& A. Amaral (Eds.), Quality Assurance in Higher Education. Contemporary Debates. (pp. 66-86). New York: Palgrave Macmillan. http://doi.org/10.1007/978-94-6091-867-4_8

Dias, D., \& Soares, D. (2016). Retrieving civic dimensions in Higher Education curricula: Is learning improving students' civic development? In EDULEARN16 Proceedings (pp. 8159-8166). http://doi.org/10.21125/edulearn.2016.0785

Dias, D., \& Soares, D. (2017). Learning outcomes in Higher Education: Designing a conceptual map for portuguese academia. In INTED2017 (pp. 91889194). Valencia: IATED. http://doi.org/10.21125/inted.2017.2170

European Commission. (2008). Improving competences for the 21st century: An agenda for european cooperation on schools. Commission Staff Working Document (Vol. 53). Brussels.

Ewell, P. (2005). Enhancing alumni research: European and American perspectives. New Directions for Institutional Research, 126, 19-29.

Gallavara, G., Hreinsson, E., Kajaste, M., \& Zadeh, M. S. (2008). Learning outcomes: Common framework different approaches to evaluation learning outcomes in the Nordic countries. Helsinki, Nordic Quality Assurance Network for Higher Education.

Garcia-Aracil, A., \& Van der Valden, R. (2008). Competencies for young European higher education graduates: Labor market mismatches and their payoffs. Higher Education, 55, 219-239.

Gonzalez, J., \& Wagenaar, R. (2003). Tuning Educational Structures in Europe. Final Report. Pilot Project - Phase 1. Deusto-Groningen, European Commission.

Joint Quality Initiative informal group. (2004). Shared "Dublin" descriptors for Short Cycle, First Cycle, Second Cycle and Third Cycle Awards. Dublin.

Moon, J. (2004). Linking evels, learning outcomes and assessment criteria. In Paper presented at the Seminar "Using Learning Outocomes (pp. 1-30). Edinburgh.

Nusche, D. (2008). Assessment of learning outcomes in Higher Education. OECD Education Working Papers, (15). http://doi.org/10.1787/244257272573

OECD. (2013). Assessment of higher education learning outcomes (AHELO): Feasibility Study Report, Vol. 3, Further Insights (Vol. 3).

Reason, R. D., \& Hemer, K. (2012). Civic learning and engagement: A review of the literature on civic learning, assessment, and instruments. Iowa State, Research Institute for Studies in Education

Scott, I. (2011). The Learning Ooutcome in Higher Education: Time to think again ? Worcester Journal of Learning and Teaching, (5), 1-8.
Shavelson, R. J., \& Huang, L. (2003). Responding responsibly to the frenzy to assess learning in higher education. Change, 31, 10-19.

Stanley, J. (2015). Learning outcomes: From policy discourse to practice. European Journal of Education, 50(4), 404-419. http://doi.org/10.1111/ejed.12150

Tissot, P. (2008). Terminology of education and training policy: A multilingual glossary. (CEDEFOP, Ed.). Luxembourg.

Tremblay, K., Lalancette, D., Roseveare, D., Dias, D., \& Amaral, A. (2013). Assessment of Higher Education learning outcomes (AHELO): Feasibility Study. Feasibility Study Report, 1, 66-86. http://doi.org/10.1007/978-94-6091-867-4_8

Veiga, A., Rosa, M. J., Dias, D., \& Amaral, A. (2013). Why is it difficult to grasp the impacts of the Portuguese quality assurance system? European Journal of Education, 48(3), 454-470. http://doi.org/10.1111/ejed.12040

Werquin, P. (2012). The missing link to connect education and employment: recognition of non-formal and informal learning outcomes . Journal of Education and Work, 25(3), 259-278. http://doi.org/10.1080/13639080.2012.687574

Wiliam, D. (2010). The role of formative assessment in effective learning environment. In OECD (Ed.), The Nature of Learning: Using Research to Inspire Practice. Paris, OECD.

\section{Agradecimientos}

Este projeto de investigação tem o apoio de Agência de Avaliação e Acreditação do Ensino Superior (A3ES). 